\title{
Georg E. Gold, Niels Juul (eds) Monitoring of cerebral and spinal haemodynamics during neurosurgery
}

\author{
Springer Verlag Berlin Heidelberg, 2008. ISBN 978-3-540-77872-1
}

\author{
Ulrich Sure • Beate Schoch
}

Received: 8 January 2009/Accepted: 12 January 2009/Published online: 13 February 2009

(C) Springer-Verlag 2009

The present book on monitoring of cerebral and spinal haemodynamics is focussed on the huge database of more than 1,800 patients of a single institution (University Hospital of Åarhus, Denmark). It describes the subdural measuring of intracranial pressure during neurosurgical surgery. In the initial chapter, a thoroughly well-written part demonstrated the present knowledge about the pathophysiology and monitoring of the intracranial pressure (ICP). It describes the various influences on ICP based on experimental, animal and human studies. In 23 additional chapters, the results of the subdural measurement of intracranial pressure under various anaesthesiological conditions and different entities are compiled by the members of the Department of Neuroanaesthesiology. All chapters are ordered like manuscripts in journals with aim, method, results and discussion. The book contains an updated bibliography with the most relevant literature and therefore represents a treasure trove for somebody, who wants to deepen the knowledge on ICP, especially measured in the subdural space. The subject index is extensive and clearly arranged.

It is noteworthy that all studies in the publication are done in a nearly identical manner: All patients received a subdural catheter after removal of the bone flap (22 G needle connected to a pressure transducer via a polyethylene catheter), a venous jugular bulb catheter and the parameters of subdural ICP, cerebral perfusion pressure, arteriovenous differences of $\mathrm{O}_{2}$ and $\mathrm{CO}_{2}, \mathrm{pH}$, lactate, $\mathrm{Na}^{+}, \mathrm{K}^{+}, \mathrm{Ca}^{++}$, cerebral blood flow and cerebral metabolic rate of oxygen using the ${ }^{133} \mathrm{Xe}$ method were measured or calculated. The majority of the patients suffered from a supra- or infratentorial tumour $(80.5 \%)$, followed by $12.3 \%$ of patients with ruptured or unruptured cerebral aneurysms. In two studies, 57 children were included. Based on the aforementioned measurements, the impact of various sedativa and analgetics (Fentanyl, Remifentanil, Propofol, Isoflurane, Sevofluran), their way of administration (bolus versus continuously) and the influence of positive end-expiratory pressure is described. Moreover, the impact of therapeutic strategies like reverse Trendelenburg, prone or supine position, anti-oedematous medication (Mannitol, Indomethacin, Dihydroergotamine), hyperventilation evacuation of cerebral cysts or surgical drainage was examined.

The value of the presented studies is limited due to the short period of monitoring of the ICP (only during the neurosurgical procedures). Unfortunately, the problems associated with the development and treatment of elevated ICP during the course of cerebral damage are not in the focus of the book. In summary, this is a book with a lot of new information on the pathophysiology of ICP that might be of interest for colleagues who are interested in a specialisation in neuroanaesthesiology. 